\title{
Cross-modal correlations of the perceived durations of auditory and visual stimuli
}

\author{
M. LOEB AND I. BEHAR, US ARMY MEDICAL RESEARCH LABORATORY, FORT KNOX, KENTUCKY
}

\section{J. S. WARM, UNIVERSITY OF LOUISVILLE}

Ratings of duration were obtained for auditory and visual signals ranging from 1 to $5 \mathrm{sec}$. The inter-modal correlations were moderately large and of an order of magnitude comparable to the intra-modal correlations. Results were considered to support the notion of mechanisms for judgment of time common to various sensory modalities.

A number of theoretical models dealing with temporal perception have postulated that judgments of duration are based upon the output of a common neural pacemaker or counting mechanism sensitive to various stimulus and organismic factors (Creelman, 1962; Hoagland, 1933; Treisman, 1963). As the pacemaker is held to be located within the central nervous system, such models demand that a degree of invariance be demonstrated between judgments of the duration of signals delivered through different peripheral channels. While previous research has generally focused upon the identification of sensory differences in temporal judgment (Behar \& Bevan, 1961; Goldstone \& Goldfarb, 1963; Hawkes, Bailey, \& Warm, 1961) the present paper is concerned with the degree of association between inter-modal judgments of signal duration. Method

The present study is based upon data previously described by Behar \& Bevan (1961). A complete description of apparatus and procedure can be found in their report. Briefly, 20 undergraduate Ss were presented, in random order, with 75 auditory and 75 visual signals of $1,2,3,4$, or 5 sec. duration. The visual signals were produced by a neon lamp (G.E. $\mathrm{NE} 51) 2 \mathrm{ft}$. in front of $\mathrm{S}$; the auditory signals were white noise, subjectively equated in intensity to that of the visual signal, fed into Permaflux PDR-8 earphones. Ss rated the duration of each stimulus on an eleven category verbal scale.

\section{Results and Discussion}

Pearson product-moment correlation coefficients were computed between median judgments given to each interval in the auditory and visual modalities. These coefficients are presented in Table 1 . Note that they range from .61 to .80 and that all are reliably greater than zero. These were converted to Fisher $\mathbf{z}$ scores and averaged (McNemar, 1962); the average

Table 1. Correlations of Ratings of Each Interval in Auditory and Visual Modalities

\begin{tabular}{ccccc}
\multicolumn{5}{c}{ Intervals (in sec.) } \\
\hline 1 & 2 & 3 & 4 & 5 \\
\hline .70 & .61 & .69 & .68 & .80 \\
\hline
\end{tabular}

All values significant beyond the .01 level. inter-modality correlation across intervals so computed was found to be .70 .

In order to determine the correlation between judgments of different intervals within a single modality, product-moment coefficients were computed between median judgments of adjacent intervals. The eight values so obtained ranged from .43 to .96 and all were significant beyond the .05 level. The mean intra-modality correlation was determined to be 0.79 for each modality. It is evident that the average inter-modality and intramodality correlations obtained here are of the same order of magnitude.

Although Behar \& Bevan's (1961) intial study reported that auditory signals are judged as longer than visual signals of equal duration, the currentanalysis indicates that inter-modal temporal judgments are highly correlated. This implies that while perception of the absolute duration of auditory and visual signals may differ, relative disparities between $S$ s in regard to judgment magnitude tend to remain invariant to a significant degree across these sensory channels.

In a recent investigation using a signal detection model, Eijkman \& Vendrik (1965) measured fluctuations in the ability of Ss to detect increments in the duration of auditory and visual stimuli. They found that with this task, the internal noise levels of the two sensory modalities were highly correlated. The data of the present study confirm and extend the findings of Eijkman \& Vendrik and provide further empirical support for the notion of common mechanisms underlying the perceived duration of stimuli presented to different sensory channels.

\section{References}

Behar, 1., \& Bevan, W. The perceived duration of auditory and visual intervals: Cross-modal comparison and interaction. Amer. J. Psychol., 1961, 74, 17-26.

Creelman, C. D. Human discrimination of auditory duration. $J$. Acoust. Soc. Amer., 1962, 34, 582-593.

Eijkman, E., \& Vendrik, J. H. Can a sensory system be specified by its internal noise? J. Acoust. Soc. Amer., 1965, 37, 11021109.

Goldstone, S., \& Goldfarb, J. L. Judgment of filled and unfilled durations: Intersensory factors. Percept. mot. Skills, 1963, 17, 763-774.

Hawkes, G. R., Bailey, R. W., \& Warm, J. S. Method and modality in judgments of brief stimulus duration. J. aud. Res., 1961, 1, 133-144.

Hoagland, $\mathbf{H}$. The physiological control of judgments of duration: Evidence for a chemical clock. J. gen. Psychol., 1933, 9, 267287.

McNemar, Q. Psychological statistics. (3rd ed.), New York: Wiley, 1962.

Treisman, M. Temporal discrimination and the indifference interval: Implications for a model of the "internal clock." Psychot. Monogr., 1963, 77, No. 13 (Whole No. 576). 